It occurred to the writer once, when faced with this dilemma, to pass two cystitomes into the lens, broadside on, if necessary, steadying the latter during the procedure by gentle pressure with a squint hook held by an assistant (vide figure). The cataract, which was not a soft one, was then easily lifted out of the eye, and no complications ensued. This manoeuvre has been repeated in two other cases with successful results, and is simpler and probably safer than the use of the vectis.

\title{
AN ATTEMPT TO TREAT A PERFORATED EYE WITH SUBCONJUNCTIVAL PENICILLIN*
}

BY

FRANK R. NEUBERT

GUERNSEY

History. September 17, 1947. Perforation of cornea by piece of wire which patient was cutting.

Examination. The eye was seen about three hours after injury. The cornea had a $3 \mathrm{~mm}$. perforation at 1 o'clock halfway between the pole and limbus. Most of the aqueous was lost. There was no iris prolapse. A thin film of whitish exudate covered the pupil and the lens could not be seen although injury of it was considered likely.

September 18,1947 . There was a sticky conjunctival discharge. The aqueous appeared slightly cloudy. No pain.

September 19, 1947. The right eye was injected. Smear and culture of L.E: shows a small growth of Gram-positive penicillinsensitive cocci. An attempt was made to save the eye by means of penicillin following the method of Sorsby and Ungar. ("Distribution of penicillin in the eye after subconjunctival injection" by Arnold Sorsby and J. Ungar, Brit. Jl. of Ophthal., September, 1947). Mydricain was injected followed by penicillin 50,000 units in adrenalin and novutox subconjunctivally. A course of intra-muscular penicillin injections 30,000 units was commenced.

September 20,1947. The corneal endothelium was almost opaque. The conjunctiva was very injected and the discharge still present. Subconjunctival injection of penicillin was repeated twice.

September 21,1947 . The conjunctival injection was increased and the cornea was quite dull. In view of the apparent deterioration the subconjunctival injections were discontinued. The intramuscular injections were continued.

\footnotetext{
* Received for publication, December 12, 1947.
} 
September 22, 1947. Two k.p. seen through the dense corneal haze. The eye was removed.

The socket was clean and healing was rapid: the man was discharged on the fifth day.

\title{
Pathological Report
}

Résumé. Corneal epithelium shed. Anterior chamber contains k.p. and fibrinous exudate. Iris, oedematous and infiltrated. Lens cataractous : capsule ruptured and early abscess formation. Abscesses present in vitreous. Retina detached and undergoing necrosis.

Diagnosis. Early panophthalmitis.

Remarks. A case is reported of an injured eye infected with penicillin-sensitive organisms which did not respond to penicillin therapy.

\section{THE USE OF ANTI-ALLERGIC DRUGS IN THE TREATMENT OF PHLYCTENULAR OPHTHALMIA *}

\author{
BY \\ C. F. Bowes \\ LONDON
}

THE striking success achieved by benadryl and allied drugs in the treatment of urticaria and a widely divided group of conditions of allergic origin, suggested that this group of drugs might give some alleviation of the photophobia, irritation, lacrimation and eczematous condition with which phlyctenular disease is associated.

\section{Subjects}

Fifteen children from $2 \frac{1}{2}$ to 11 years of age. All had clinical evidence of phlyctenular disease, and showed positive Mantoux tests, and X-ray evidence of tuberculous infection; 9 cases had a family history of tuberculosis and 8 were contacts). In all all cases the drug concerned was given when the symptoms were particularly troublesome and hence any improvement would have been particularly noticeable.

\section{Drugs used}

The two drugs used were benadryl (Parke Davis and Co. Ltd.), and antistin (Ciba). $\therefore$ In 7 cases both agents were employed after an interval of at least one week.

\footnotetext{
* Received for publication, November 25, 1947.
} 